\title{
The Multiple Shell PN NGC 2438: Shell Modeling and the Influence of Different Central Star Models
}

\author{
Birgit Armsdorfer \& Stefan Kimeswenger \\ Institut für Astrophysik, Leopold-Franzens-Universität Innsbruck, \\ Technikerstr. 25, A-6020 Innsbruck, Austria \\ Thomas Rauch \\ Institut für Astronomie und Astrophysik, Universität Tübingen, Sand 1, \\ D-72076 Tübingen, Germany
}

\begin{abstract}
Modeling the shells of multiple shell planetary nebulae using different model spectra for hot central stars, we found that a blackbody model leads to wrong nebular parameters. We model the density profile of the outer shells, varying the results of hydrodynamical simulations. This leads to a spatial excitation profile which reproduces well the observations.
\end{abstract}

We investigate the influence of different input parameters of model spectra for hot central stars (CSPNe) on the emission of evolved multiple shell planetary nebulae $(\mathrm{PNe})$. As an example, we present the spherical multiple shell PN NGC 2438 which has a well modeled central star and regularly shaped shells (Rauch et al. 1999; Rauch 2001).

For our studies, we combined central star atmosphere modeling (ranging from blackbody flux to NLTE atmosphere models with different element coverage, i.e. $\mathrm{H}-\mathrm{He}, \mathrm{H}-\mathrm{Ca}$, and $\mathrm{H}-\mathrm{Ni}$ composition; Rauch 2001) and nebular shell modeling to obtain self consistent presentations of the nebulae. The stellar fluxes were calibrated to have the same visual magnitude, effective temperature $\left(T_{\text {eff }}=110 \mathrm{kK}\right)$, and surface gravity $(\log g=7(\mathrm{cgs}))$. Geometrical parameters of the nebula were derived from narrow-band images and long-slit spectra.

Due to interstellar gaseous absorption below $912 \AA$, observational insight into hot CSPNe is not possible in the region of maximum interest for such stars. PNe spectra strongly reflect the UV spectra of those stars in the optical region. Therefore, modeling of optical nebular spectra serves as a valuable proof for the theoretical modeling of CSPNe in the UV.

As a first step, we examined the effects of different model spectra on the modeling of the bright inner rim of the nebula, using a density profile similar to the one given by Corradi et al. (2000) but with a filling factor of 0.1 . The photo-ionization code Cloudy90 (Ferland 1996) was applied to model the emitted spectrum of the nebula (Armsdorfer, Kimeswenger, \& Rauch 2001). We found that hydrogen lines are nearly unaffected by the different illumination sources, while other lines change both in strength and spatial profile (this might also be the reason why the use of a blackbody model was presumed to be sufficient when only hydrogen was tested extensively). For the low ionized species, the blackbody overestimates the lines from $30 \%$ ([S II]) to $45 \%$ ([N II]) compared to the $\mathrm{H}-\mathrm{Ni}$ model atmosphere. For the [O III] lines, it underestimates the real 

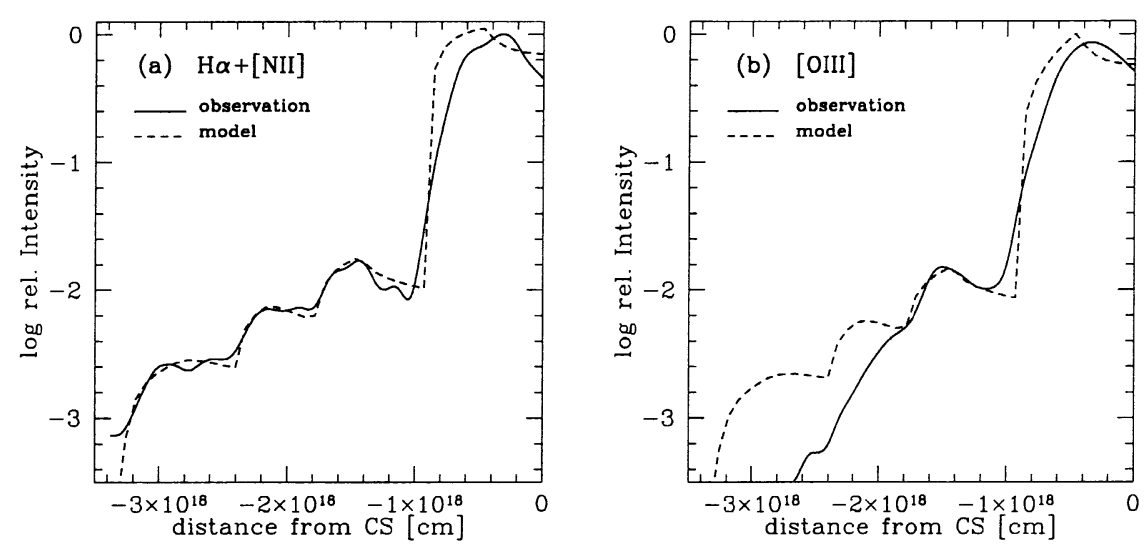

Figure 1. Comparison of the observed and modeled surface brightness in (a) $\mathrm{H} \alpha+[\mathrm{N}$ II] and (b) [O III] $(\lambda 4959+5007 \AA)$ of NGC 2438. The different shells are clearly visible. We varied the filling factor to reproduce the optical spectral behaviour of the outer shells where we still have a significant amount of ionizing UV photons. The excitation class (Gleizes, Acker, \& Stenholm 1989) of the rim of the main nebula and of the first shell is identical, both in the model and in the observations.

flux by $30 \%$. He I $(\lambda 4471 \AA)$ is underestimated by $20 \%$, while He II $(\lambda 4686 \AA)$, which shows the strongest spatial variations, is overestimated by $270 \%$. When compared to older models, the He emission shows the strongest changes in intensity and shape with the introduction of the latest state-of-the-art NLTE model atmospheres including the iron group elements. These changes strongly affect classical diagnostic diagrams and CSPN parameters obtained from them.

The investigation of the excitation of the multiple outer shells of this PN (see Fig. 1) was carried out using the H-Ni NLTE model atmosphere for the CSPN, and as a first order approximation for the electron density profile results from hydrodynamical simulations of shell evolution (Schönberner \& Steffen 2001). To obtain shells excited by the radiation field corresponding to our observational data, we had to change this density profile up to one order of magnitude.

\section{References}

Armsdorfer, B., Kimeswenger, S., \& Rauch, T. 2001, RevMexAA, in press

Corradi, R.L.M., Schönberner, D., Steffen, M., \& Perinotto, M. 2000, A\&A, 354, 1071

Ferland, G. J. 1996, Hazy, a Brief Introduction to Cloudy 90.05, Univ. Kentucky, Department of Physics and Astronomy, Internal Report

Gleizes, F., Acker, A., \& Stenholm, B. 1989, A\&A, 222, 237

Rauch, T. 2001, RevMexAA, in press

Rauch, T., Köppen, J., Napiwotzki, R., \& Werner, K. 1999, A\&A, 347, 169

Schönberner, D. \& Steffen, M. 2001, RevMexAA, in press 